\title{
Viscoelastic Properties of Deep-Quenched Polymer Blends under Steady Shear Flow
}

\author{
Yoshiaki TaKahashi, Shinichi Kitade, Kenji OchiaI, and Ichiro Noda \\ Department of Applied Chemistry, Graduate School of Engineering, \\ Nagoya University, Nagoya 464-01, Japan
}

(Received January 29, 1997)

\begin{abstract}
KEY WORDS Viscoelastic Properties / Immiscible Polymer Blend / Polystyrene / Poly(2-chlorostyrene) / Deep Quench / Steady Shear Flow / Shear Stress / First Normal Stress Difference /
\end{abstract}

In previous papers, we reported viscoelastic properties of immiscible polymer blends in two extreme experimental conditions, i.e., in completely immiscible regions and near the critical point of the phase diagram. The former was studied by using polydimethylsiloxane (PDMS)/polybutadiene (PB) ${ }^{1}$ and PDMS/polyisoprene $(\mathrm{PI})^{2}$ blends made of the components with almost the same viscosity, and the latter was studied by shallow quenched polystyrene (PS)/poly(vinyl methyl ether) (PVME) $)^{3}$ blends $\left(\Delta T=T-T_{\mathrm{cl}}<13^{\circ} \mathrm{C} ; T_{\mathrm{cl}}\right.$ is the cloud point). Both systems satisfy the condition that the viscosities of two phases are almost the same since the two phases near the critical point also have almost the same viscosity, though these systems exist in quite different regions in the phase diagram. Therefore, all the experimental results can be discussed in comparison with the theories of Doi-Ohta ${ }^{4}$ and Onuki, ${ }^{5}$ which were originally derived for the completely immiscible binary fluids mixtures and the near-critical phase-separated system, respectively, since both theories give the same prediction that the excess stresses of blends of two immiscible fluids with the same viscosity are proportional to shear rate $\dot{\gamma}$ and viscosity of components owing to the interfacial tension of deformed domains interface.

It was observed for completely immiscible PDMS/PB ${ }^{1}$ and PDMS $/ \mathrm{PI}^{2}$ blends that both shear stress $\sigma$ and first normal stress difference $N_{1}$ are proportional to $\dot{\gamma}$ (textured materials' behavior) as predicted by the theories. ${ }^{4,5}$ For PS/PVME ${ }^{3}$ blends near the critical point, on the other hand, the textured materials' behavior was observed as expected, but the $\dot{\gamma}$ range of textured materials' behavior was rather narrow because the flow induced homogenization occurred at a critical shear rate $\dot{\gamma}^{* *}$, so that $N_{1}$ became almost proportional to $\dot{\gamma}^{2}$ as expected for homogeneous systems ${ }^{6}$ at $\dot{\gamma}>\dot{\gamma}^{* *}$. As $\dot{\gamma}^{* *}$ became slightly higher with increasing quench depth, it can be expected that the textured materials' behavior is observed in the wider $\dot{\gamma}$ region at the deep quench condition as long as the viscosities of two phases are almost the same. It is also interesting to examine whether the flow induced homogenization occur or not at the deep quench condition. However, these study cannot be performed for PS/PVME systems since the viscosities of two phases become quite different at the deep quench condition.

In this work, therefore, we study viscoelastic properties of another immiscible polymer blend at the deep quench condition under steady shear flow, where the separated phases have almost the same viscosity. The system used is a PS and poly(2-chlorostyrene) (P2ClS) blend, which has an almost symmetric LCST type phase diagram ${ }^{7,8}$ and the separated phases are expected to have similar viscosities.

\section{EXPERIMENTAL}

Polymer samples used are a standard PS F10 (nominal $M_{w}=9.64 \times 10^{4}$ and $\left.M_{w} / M_{n}=1.01\right)$ of Tosoh Co., Ltd., and a P2ClS of Scientific Polymer Products Inc. The former was used as received, while the latter was fractionated a few times in benzene-methanol $8: 2$ mixed solvent. $M_{w}$ and $M_{w} / M_{n}$ of fractionated P2ClS measured by GPC-LALLS of Tosoh was $12.3 \times 10^{4}$ and 1.27, respectively. Dibuthylphthalate (DBP) was added to the polymer blends $(5.8 \mathrm{wt} \%)$ to lower the phase separation temperature $^{7,8}$ and also lower the viscosity. It was also added to the component polymers for comparison.

Homogeneous and transparent films of component samples PS/DBP (5.8 wt \%), P2ClS/DBP (5.8wt \%), and blend samples PS/P2CIS/DBP (5.8 wt \%) were prepared for cloud point or viscoelastic measurements by solvent cast method, following the procedure of Okada, et al.$^{7,8}$

Cloud points of blend samples with different compositions were determined by heating the sample with the rate of $0.1^{\circ} \mathrm{C} \mathrm{min}^{-1}$ and by measuring the transmitted intensity of a $\mathrm{He}-\mathrm{Ne}$ laser through a $0.2 \mathrm{~mm}$ thick sample film. ${ }^{3}$

Viscoelastic properties of the blend sample at the critical composition were measured at 130, 140, and $150^{\circ} \mathrm{C}$ under steady shear flows with a Rheometrics Mechanical Spectrometer RMS800 with cone and plate geometries with 0.1 rad cone angle and either 7.9 or $25 \mathrm{~mm}$ diameter. Viscoelastic properties of the components were also measured with the RMS800 under oscillatory shear flow at various temperatures and the master curves of storage and loss moduli were generated by the usual procedure. Zero shear viscosities of the component samples at 130,140 , and $150^{\circ} \mathrm{C}$ evaluated from the master curve are tabulated in Table I.

\section{RESULTS AND DISCUSSION}

Figure 1 shows a cloud point curve of PS/P2CIS/DBP blends. It is clear from this figure that the phase diagram 


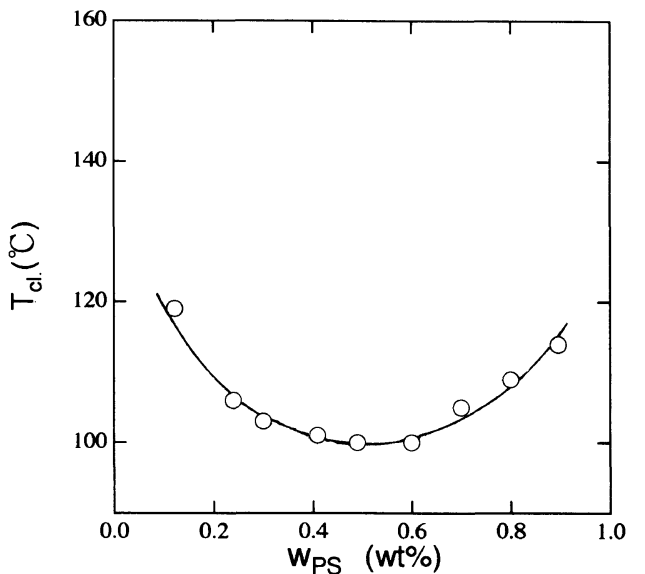

Figure 1. Composition dependence of cloud point $\left(T_{\mathrm{c} 1}\right)$ of PS/P2ClS/ DBP blends. $W_{\mathrm{PS}}$ denotes the wt $\%$ of PS in the total polymers.

Table I. Zero shear viscosities of component polymers

\begin{tabular}{lccc}
\hline \multirow{3}{*}{ Sample } & \multicolumn{3}{c}{$\eta^{0} / \mathrm{Pa} \mathrm{s}^{-1}$} \\
\cline { 2 - 4 } & $130^{\circ} \mathrm{C}$ & $140^{\circ} \mathrm{C}$ & $150^{\circ} \mathrm{C}$ \\
\hline PS/DBP & $7.0 \times 10^{4}$ & $1.9 \times 10^{4}$ & $3.4 \times 10^{3}$ \\
P2ClS/DBP & $9.0 \times 10^{4}$ & $1.8 \times 10^{4}$ & $5.2 \times 10^{3}$ \\
\hline
\end{tabular}

is almost symmetric, and the critical composition and temperature are $50 \mathrm{wt} \%$ and $100^{\circ} \mathrm{C}$, respectively. Thus the composition of the blend sample for viscoelastic measurements were fixed at $50 \%$ to maintain almost the same symmetrical composition ratio in the two phases separated by deep quench. As it can be seen in Figure 1 , the compositions of minor components in the separated phases are very low for the present deep quench conditions. Since the two component homopolymers have the similar viscosities (Table I), we can safely assume that the two phases have almost the same viscosities over a wide range of temperature.

Figure 2 shows double logarithmic plots of $\sigma$ and $N_{1}$ against $\dot{\gamma}$ for the blend samples. It is clear in Figure $2 b$ that $N_{1}$ is proportional to $\dot{\gamma}$ in the measured $\dot{\gamma}$ region and the region is wider in these deep quench condition than near the critical point in a previous paper, ${ }^{3}$ as expected. No shear induced homogenization was observed in the measured $\dot{\gamma}$ range, which was detected by the change in the $\dot{\gamma}$ dependence of $\mathrm{N}_{1}$ in PS/PVME blends near the critical point. ${ }^{3}$

It is also clear in Figure 2a that $\sigma$ is proportional to $\dot{\gamma}$ at low $\dot{\gamma}$ region, but the proportionality becomes lower at the higher $\dot{\gamma}$ region at all temperatures. Moreover, there exists a small shoulder or plateau in the middle range of $\dot{\gamma}$. To discuss these behavior further, the shear viscosity $\eta(=\sigma / \dot{\gamma})$ at $130^{\circ} \mathrm{C}$ is plotted against $\dot{\gamma}$ in Figure 3 . The figure also shows the dynamic viscosities of the component samples, $\eta(\omega)$, which almost represents the $\dot{\gamma}$ dependence of $\eta(\dot{\gamma})$ for homopolymers, ${ }^{6}$ for comparison.

It is observed that $\eta(\dot{\gamma})$ of the blend sample is almost constant at the low $\dot{\gamma}$ region where the viscosities of the components are almost the same and show only slight shear thinning. The viscosity of the blend is about twice larger than those of the components at this region. The viscosities of the two components decrease with increasing shear rate $\dot{\gamma}$. The viscosities for each com-
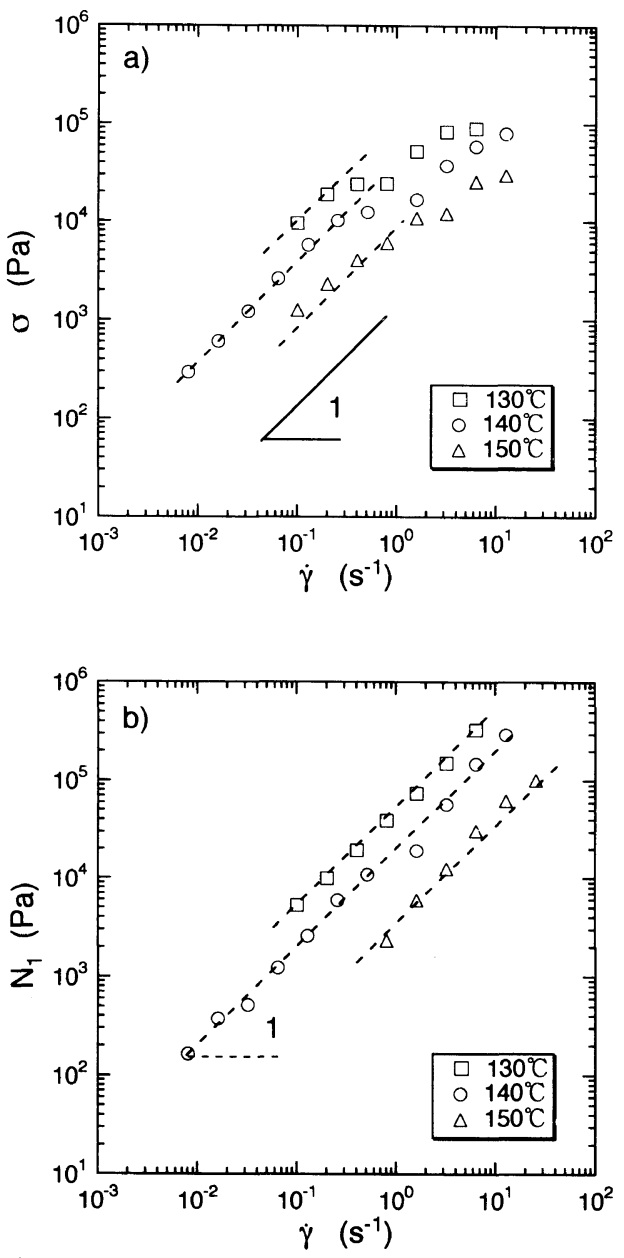

Figure 2. Shear rate dependence of a) shear stress and b) first normal stress difference of a PS/P2ClS/DBP blend $\left(W_{\mathrm{PS}}=50 \%\right)$ at 130,140 , and $150^{\circ} \mathrm{C}$.

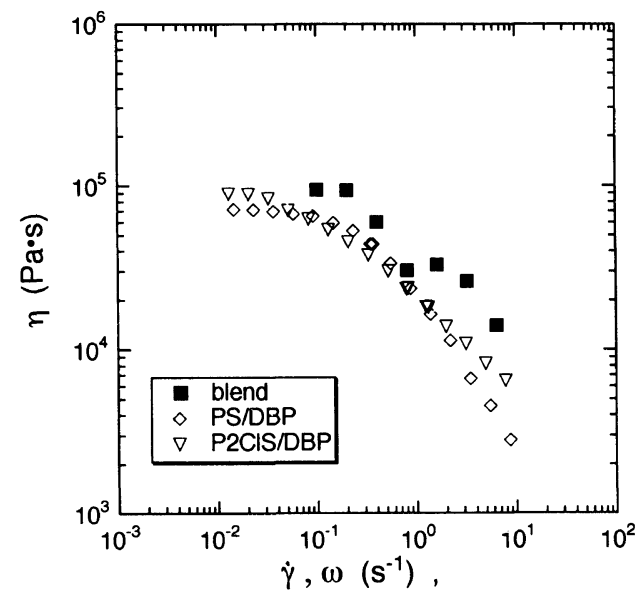

Figure 3. Shear rate dependence of shear viscosity of PS/P2C1S/DBP $\left(W_{\mathrm{PS}}=50 \%\right)$ blend, together with dynamic viscosities of PS/DBP and $\mathrm{P} 2 \mathrm{ClS} / \mathrm{DBP}$, at $130^{\circ} \mathrm{C}$.

ponent decrease in slightly different manner and cross each other at a certain $\dot{\gamma}$. This may be due to the fact that the PS component is a nearly monodisperse sample and it is entangled, while the P2ClS component has relatively broad molecular weight distribution and it is hardly entangled. ${ }^{6}$ From these results, we speculate that the $\dot{\gamma}$ dependence of $\eta$ of the blend sample reflect that of the component having the higher viscosity, so 
that a small shoulder or plateau appears in the middle range of $\dot{\gamma}$.

Since $N_{1}$ is proportional to $\dot{\gamma}$ in the measured $\dot{\gamma}$ range, on the other hand, the contribution from the components seems to be negligible. At the high $\dot{\gamma}$ end, the components will somewhat contribute to the observed $N_{1}$, but the excess $N_{1}$ will decrease in this region reflecting the shear thinning of the viscosities of the components. These two effects could cancel each other, so that the linear proportionality of $N_{1}$ with $\dot{\gamma}$ for the blend may be observed in the much wider range of $\dot{\gamma}$. These speculations should be experimentally examined by studying viscoelastic properties of immiscible blends in the non-Newtonian region in more details. At present we conclude that the viscoelastic properties of binary polymer blends in the phase separated regions, from very close to the phase transition point to completely immiscible cases, can be basically explained by the theories of Doi-Ohta ${ }^{4}$ and
Onuki, ${ }^{5}$ as long as the two phases have almost the same viscosities.

\section{REFERENCES}

1. Y. Takahashi, S. Kitade, N. Kurashima, and I. Noda, Polym. J., 26, 1206 (1994).

2. Y. Takahashi and I. Noda, in "Flow-Induced Structures in Polymers," A. I. Nakatani and M. D. Dadmun, Ed., ACS Symposium Series 597, American Chemical Society, Washington, D.C., 1995.

3. Y. Takahashi, H. Suzuki, Y. Nakagawa, and I. Noda, Macromolecules, 27, 6476 (1994).

4. M. Doi and T. Ohta, J. Chem. Phys., 95, 1242 (1991).

5. A. Onuki, Phys. Rev. A, 35, 5149 (1987).

6. W. W. Graessley, Adv. Polym. Sci., 16, 1 (1974).

7. K. D. Kwak, M. Okada, and T. Nose, Polymer, 32, 864 (1991).

8. K. D. Kwak, M. Okada, T. Chiba, and T. Nose, Macromolecules, 26, 4047 (1993). 